\title{
The direct diagnosis of myocardial infarction by photoscanning after administration of cesium-131
}

\author{
Edward A. Carr, Jr., M.D. \\ Geoffrey Gleason, B.Ch.E. \\ James Shaw, A.B. \\ Brian Krontz \\ Ann Arbor, Mich.
}

$\mathrm{I}^{\mathrm{n}}$ view of the clinical importance of myocardial lesions, a technique for visualizing them in the intact patient is clearly desirable. The advent of scintillation scanning suggested a promising approach to this problem. The first encouraging result was the finding that dogs with experimental myocardial infarcts showed less concentration of rubidium- 86 in the area of infarction than in normal myocardium. ${ }^{1}$ This permitted clear visualization of the infarct if the heart were subsequently removed from the $d o g$, but the high energy of the gamma emission of rubidium-86 made it unsuitable for studies of the heart in situ. The next step, demonstration of experimental myocardial infarcts through the intact chest wall of the living animal, was first successfully accomplished by the use of mercury-203labeled chlormerodrin. ${ }^{2}$ When applied to human beings, however, this technique proved to be only irregularly successful. ${ }^{3}$ The development of a method for obtaining suitable quantities of cesium-131 for use in human beings has now permitted the use of the latter isotope in myocardial scanning. ${ }^{3}$ The present communication demonstrates the usefulness of cesium-131

in experimental myocardial infarcts in dogs and in myocardial infarcts in human beings.

\section{Methods}

Preparation of cesium-131. Cesium-131 was prepared in a nuclear reactor by neutron irradiation of barium. Barium-130, a stable isotope present in natural barium to the extent of only 0.1 per cent, reacts to produce barium-131, which in turn decays to produce cesium-131.

$\mathrm{Ba}^{130}(\mathrm{n}, \gamma) \mathrm{Ba}^{131} \frac{\mathrm{e}, \gamma}{\mathrm{T} 1 / 2=11.6 \text { days }} \rightarrow \mathrm{Cs}^{131}$

Despite the low isotopic abundance of barium-130, the combination of a ten barn cross-section of the isotope and a high flux irradiation makes it possible to produce reasonable amounts of cesium-131. As barium-131 decays with a half life of 11.6 days to cesium-131, the former isotope may be reprocessed periodically for the extraction of cesium-131.

Cesium was separated from the cesiumbarium mixture by the use of a weakly acidic carboxylate-type cation exchanger in the ammonium form. Barium exhibits a high affinity for this exchanger when ap-

Frum the Departments of Internal Medicine (Nuclear Medicine Unit) and Pharmacology, the Kresge Research Radioisotope Unit, University of Michigan, Ann Arbor, Mich., and the Abbott Laboratories, Oak Ridge, Tenn.

This study was aided in part by grants from the Michigan Memorial Phoenix Project and the Atomic Energy Commission.

Received for publication Feb. 25, 1964. 
plied at a pH of 6 to 7. Cesium was then eluted, using a $0.15 \mathrm{~N}$ ammonium acetate buffer at $\mathrm{pH}$ 6. Cesium-131 has a half life of $9.71 \pm 0.05$ days and decays exclusively by orbital electron capture directly to the ground state of xenon-131. The $29.4 \mathrm{kev}$. xenon $\mathrm{x}$-ray resulting from $\mathrm{K}$ capture is the only radiation detectable with conventional sodium-iodide detectors. The absorption of the $29.4 \mathrm{kev}$. $\mathrm{x}$-ray in water has a half value of approximately 2.4 centimeters. Cesium-131 was administered to patients and experimental animals in the form of a sterile, carrier-free solution of the sulfate, chloride, or acetate, adjusted for isotonicity by the addition of sodium chloride.

Studies in dogs. Twenty-seven dogs received cesium-131 intravenously in doses of 40 to $650 \mu \mathrm{c}$. In preliminary work, 8 additional dogs received 250 to $500 \mu \mathrm{c}$ of cesium-134 intravenously. Of the 35 dogs studied, 15 were used in preliminary work for information on scanning techniques, tissue uptake, etc. Each of the other 20 (10 dogs subjected to coronary artery ligation, 3 control dogs with sham operations, and 7 dogs without operations) underwent complete studies, according to the protocol previously described. ${ }^{2}$ In this protocol, experimental myocardial infarcts were produced by ligation of the anterior descending branch of the left coronary artery. After closure of the chest and recovery, the dogs were studied 1 to 57 days after operation. At the time of study, each dog received cesium-131 and was scanned in the supine position at intervals ranging from 1 hour to 3 hours after injection of the isotope. A commercial scanner* with a 19-hole focusing collimator and 3 -inch crystal was used. The focus point of the collimator was $7 \mathrm{~cm}$. from its face. At the conclusion of the scan, the animal was sacrificed and the heart transfixed by long needles driven through the anterior chest wall. The anterior chest wall was removed and the exact projection of the cardiac outlines placed on the scan. The heart was then excised, emptied of blood, and rescanned. Sections of tissue from liver, myocardium, and other thoracic organs were then taken for the determination of radio- activity in a well counter. Myocardial sections were subsequently sent to a pathologist for histologic examination; whereever subsequent reference is made to infarcted or normal canine myocardium, the status of the tissue has been verified in each case by microscopic examination. Thus, except for the use of radioactive cesium in the present studies, the protocol followed in detail that previously described for the use of mercury-203-chlormerodrin.

Studies in man. On the basis of the results obtained in dogs, 13 patients received 1.1 to $2.5 \mathrm{mc}$. of cesium-131 intravenously; in the majority the dose was $1.25 \mathrm{mc}$. The estimated radiation dose (whole body) was 400 millirads. Two and three-quarters hours after injection, each patient was scanned in the supine position, using another scanner of the same model as that employed in dogs. The 19-hole collimator was placed approximately $1.5 \mathrm{~cm}$. above the chest wall. Before scanning took place, the precordial area was scouted by manually moving the probe over various parts of the heart to establish the point of maximum count rate. Except in the case of a posterior infarct, this area was usually found 5 to $6 \mathrm{~cm}$. above the xiphoid and about $3 \mathrm{~cm}$. to the left of the mid-sternal line. It was necessary to exercise care in order to avoid confusing the high count rate obtained over the liver with counts from the myocardium.

After the maximum myocardial count rate had been obtained, the scanner voltage was set to produce maximum density of the film at this count rate. The scanner was adjusted for sharp contrast.* Scans were begun at the xiphoid and were extended cephalad to approximately the lower level of the second rib. The field of the scan extended laterally from the body wall on the left side to a line approximately $7 \mathrm{~cm}$. to the right of and parallel to the mid-sternal line. By observation of the dot scan, it was usually possible to readjust the lateral margins of the scan after the first several lines had been recorded, bringing the margins in and permitting faster completion of the scan. Scans were usually

\footnotetext{
*With the Picker scanner, the "count rate differential" knob was set at 15 . With certain other scanners, an analogous setting would be a "suppression" setting of approximately
} 85. 


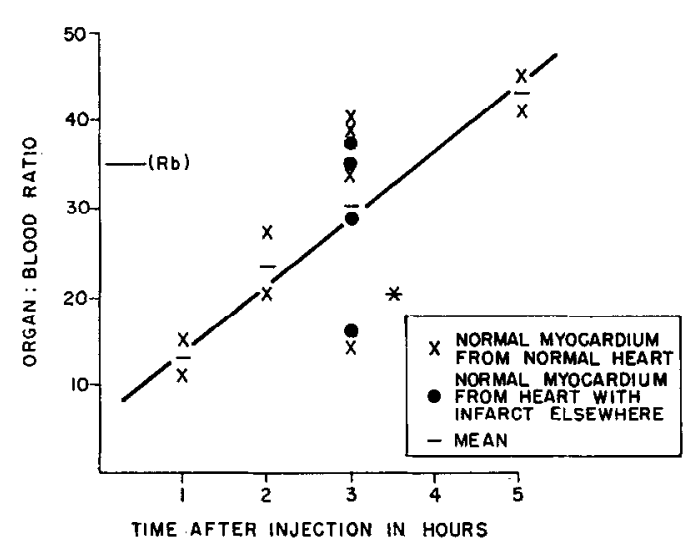

Fig. 1. Uptake of cesium-131 by normal canine myocardium. Each point represents normal myocardium from an individual dog. The organ:blood ratio is the ratio, [cesium-131] in myocardium/[cesium-131] in blood of the same animal. Note that the dots refer to normal myocardium from hearts having infarcts elsewhere in them, whereas the other points represent myocardium from entirely normal hearts.

performed at a speed of $16 \mathrm{~cm}$. per minute, with a spacing of $0.45 \mathrm{~cm}$. between lines. The scans took approximately 50 minutes to complete. Patients were brought in their beds to the Nuclear Medicine Unit and scanned without the necessity of moving from their beds at any time. When each patient had subsequently recovered sufficiently from his infarct to permit chest $x$-ray examination, a film was taken and the cardiac silhouette projected onto the scan, with appropriate corrections for difference in focus. Since the heart $x$-ray film was taken with the patient in the upright position, the projection of the cardiac outline on the scan could not be exact. Although not necessary for the reading of the scan, this crude method of superimposition made orientation of the scan somewhat easier.

The 13 patients were classified before scanning as follows: (a) 6 had suffered unequivocal acute myocardial infarction 2 to 16 days previously; (b) 2 had suffered probable recent acute myocardial infarction; (c) 2 had heart disease but had probably not suffered recent myocardial infarcts; (d) 3 were control patients without any evidence of heart disease. Each of the 6 patients with unequivocal recent infarction met all three of the following criteria: a classic history of recent acute infarction, clearly diagnostic electrocardiographic changes, and elevation of serum glutamic oxaloacetic transaminase or lactic dehydrogenase activities above normal. Each of the 3 control patients met all of the following criteria: no history suggestive of heart disease, no physical findings indicating heart disease, normal appearance of the heart on an $x$-ray film of the chest, and normal electrocardiogram. Each control patient was over 40 years old, thus placing the controls in the same general age group as the patients with acute infarction. The 4 patients in the two intermediate classes (Groups b and c) are further described below.

\section{Results}

Dogs. The normal myocardium of the dog showed a rapid uptake of radioactive cesium. Two hours after injection, the myocardium contained 20 times as much radioactivity per gram as whole blood. Normal myocardium from dogs with an infaret elsewhere in the heart showed no significant difference from normal myocardium obtained from entirely normal hearts (Fig. 1). However, these studies were designed primarily to provide a background for developing a scanning technique; the more careful clearance studies of Levy ${ }^{4}$ have suggested that ligation of a branch of the coronary artery increases the uptake of rubidium- 86 by areas of myocardium supplied by unoccluded vessels. No other thoracic tissue showed sufficient

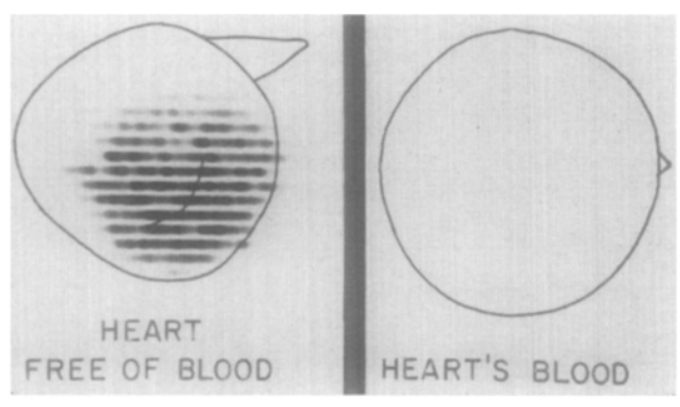

Fig. 2. Absence of detectable radioactivity in heart's blood scanned simultaneously with the heart from which it had been taken. On the left the cesium-131 in the myocardium is clearly delected by the photoscan, whereas the entire content of heart's blood, emptied into a beaker, is not visualized. Both the emptied heart and beaker of blood were scanned simultaneously, the scanner settings being the same for both. 


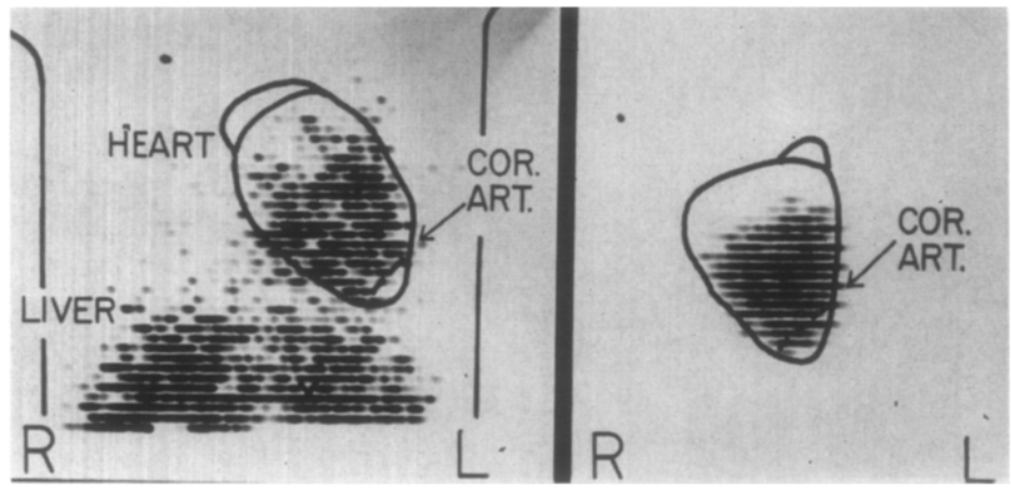

Fig. 3. Normal canine myocardial scan. On the left, the scan of the beating heart of the living dog through the intact chest wall. On the right, the isolated heart of the same dog, empticd of blood and rescanned. That portion of the anterior descending branch of the left coronary artery that was visible when the chest was opened is shown in projection on the scan.

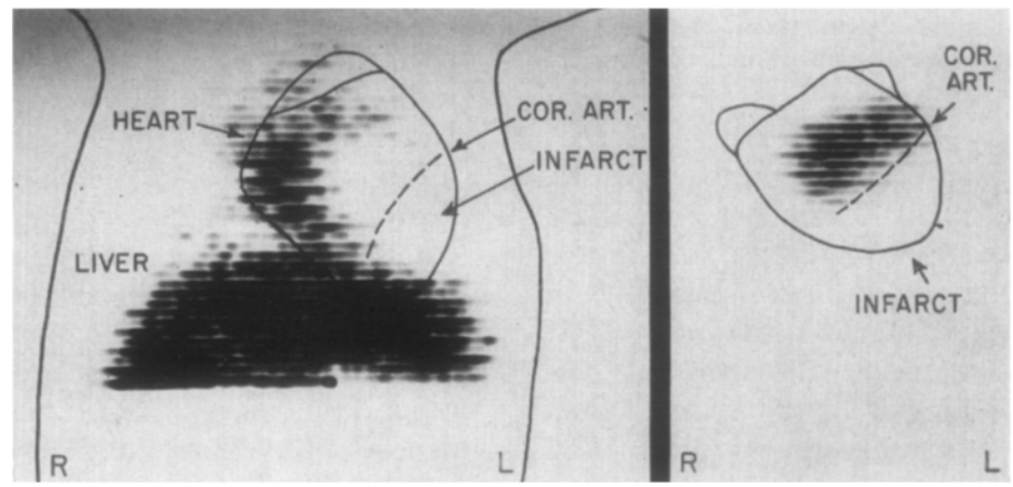

Fig. 4. Extensive anterior myocardial infarct in a dog. On the left, the scan of the intact dog shows a large "cold" area, corresponding to the infarct. This is confirmed by the scan of the isolated heart, on the right. There was extensive necrosis in the area indicated by the scan.

uptake of cesium to interfere with the myocardial scan. However, the liver showed appreciable uptake, and the ratio of hepatic uptake to myocardial uptake increased with time. The optimum time between injection of cesium-131 and performance of a myocardial scan is, therefore, a compromise between two factors: the longer the time between injection and scanning, the higher the myocardial uptake, relative to the blood; the shorter the time between injection and scanning, the higher the myocardial uptake, relative to the liver. The optimum time to begin the scan appeared to be 2 to 3 hours after injection. At that time the concentration of radioactive cesium in the myocardium is ap- proximately twice that in the liver and approximately 20 times that in the blood. The latter value should permit demonstration of the myocardium without detection of any radioactivity from the blood contained within the heart cavity. As a further proof of the latter point, one dog was sacrificed one and two-thirds hours after the injection of radioactive cesium, and all the great vessels to the heart were clamped, thus trapping within the heart the entire diastolic contents of the heart cavities. A needle was then introduced through the myocardium, and all the blood was evacuated from the heart. This entire volume of blood was placed in a beaker. The emptied heart and the beaker of 
blood were then scanned in a single scan, the same scanning factors applying to each. The result (Fig. 2) showed a clear outline of the empty left ventricle, whereas the beaker of blood gave no detectable radioactivity with this technique.
The normal left ventricular myocardium of the dog was well delineated in scans taken $21 / 2$ hours after the administration of 150 to $650 \mu \mathrm{c}$ of cesium-131. Fig. 3 shows such a normal scan. On the left, the scan obtained from the beating heart

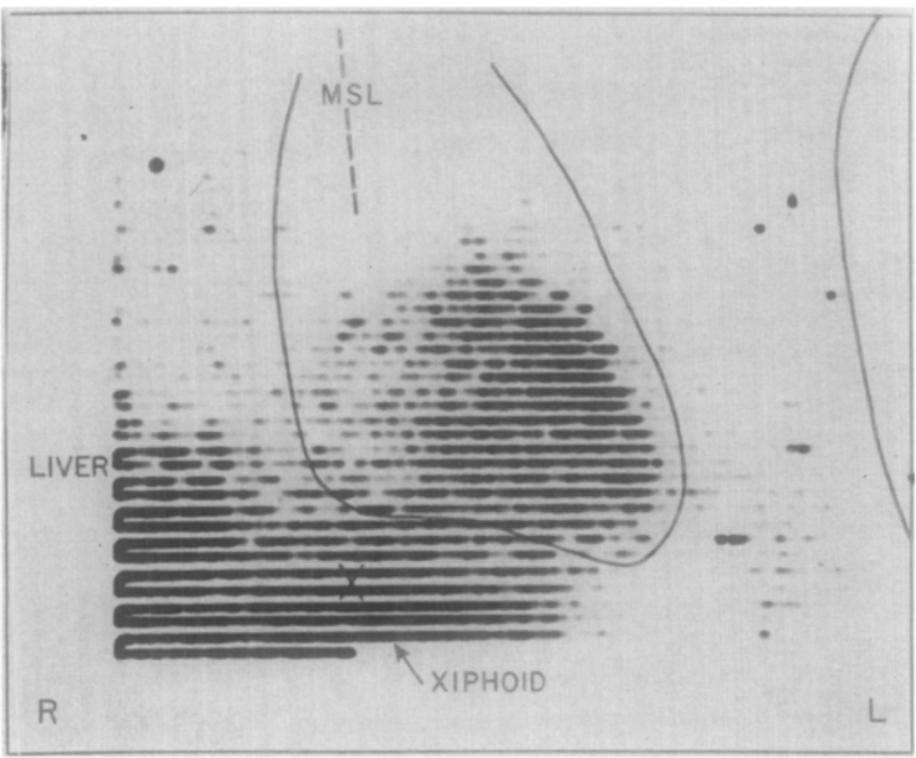

Fig. 5. Normal myocardial scan in a 52-year-old man. The left ventricle is visualized through the thinner, overlying right ventricle; the latter is not visualized because of its lesser mass. The outline of the heart shadow was projected onto the scan from a separately taken x-ray film. $M S L$ : Mid-sternal line.

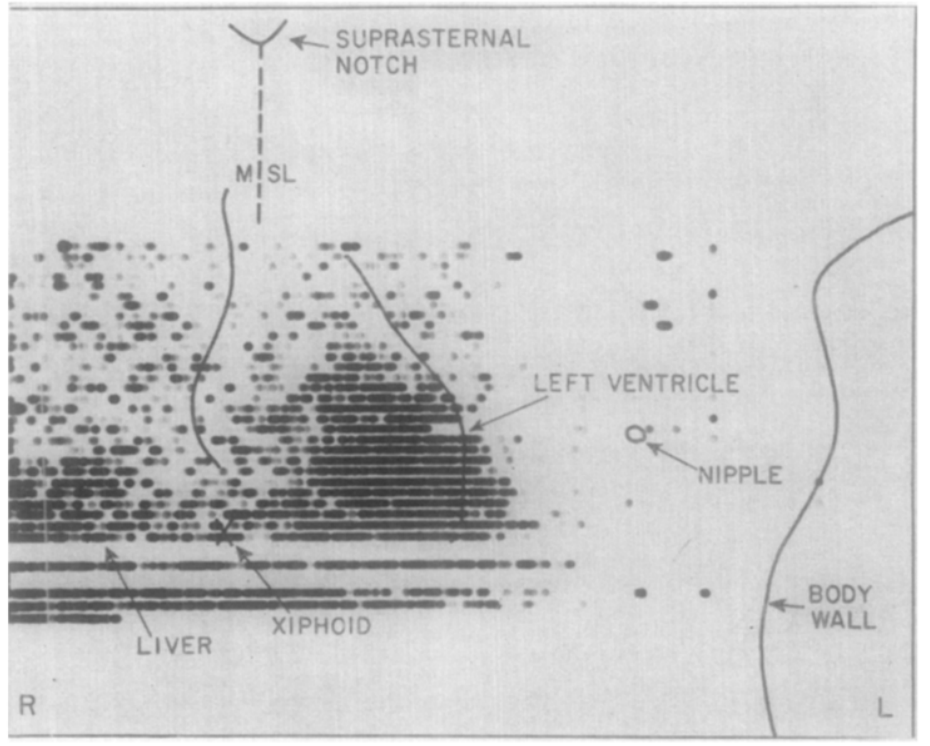

Fig. 6. Normal myocardial scan in a 61-year-old woman. 


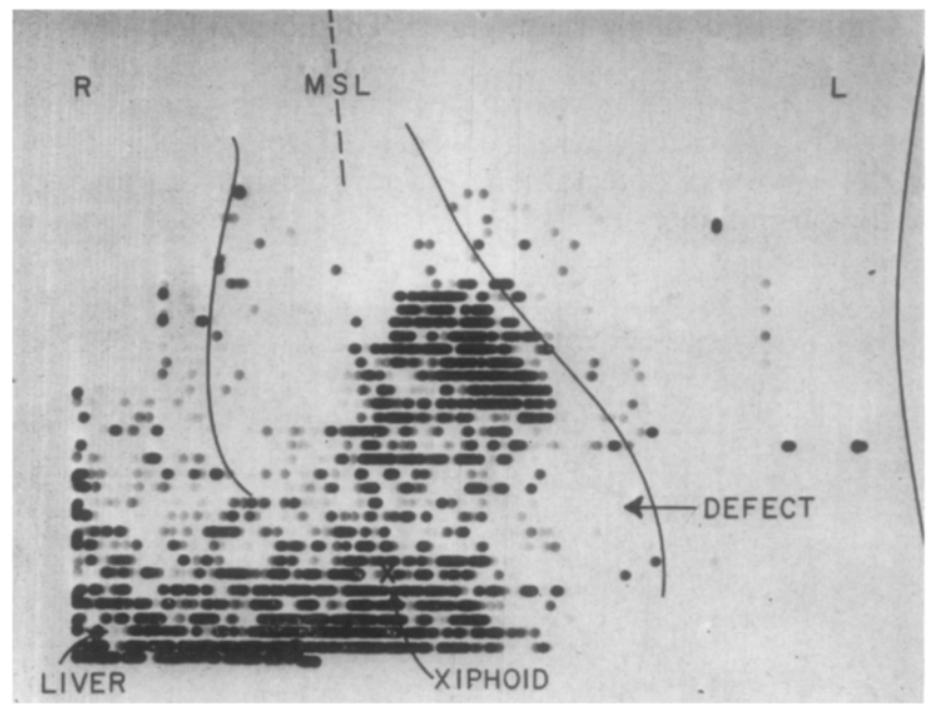

Fig. 7. Anterior myocardial infarct in a 59-year-old man, scanned 3 days after the acute episode of infarction. (In Figs. 7 through 11, the stated location of the infarct is that demonstrated by the electrocardiogram.)

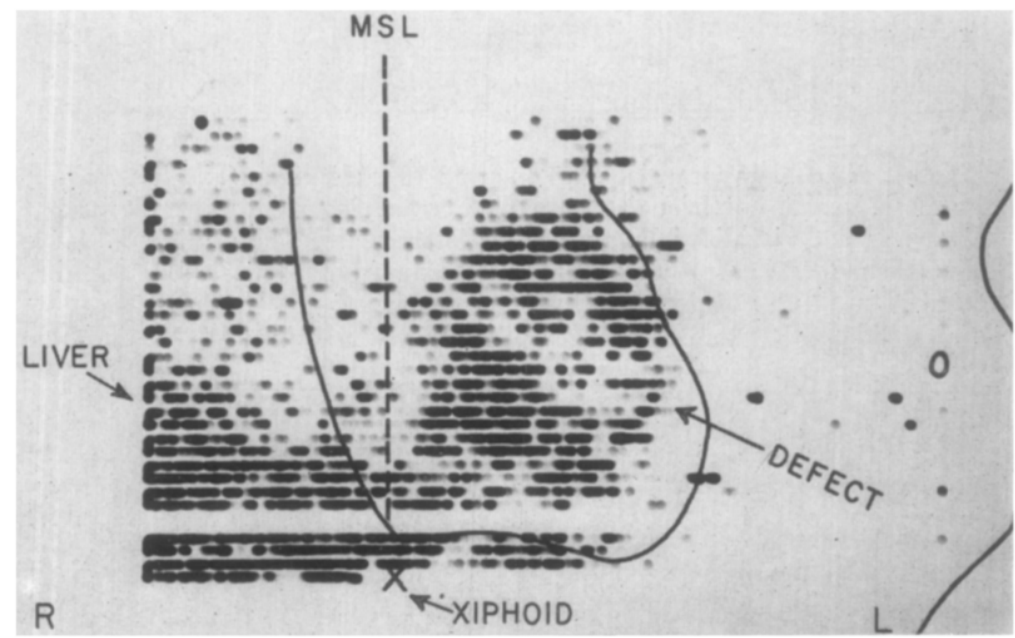

Fig. 8. Anteroseptal infarct in a 66-year-old woman, scanned 2 days after the acute episode.

of the living dog through the intact chest wall is shown. The full, even density of the left ventricle appears through the thinner overlying right ventricular wall. Although the concentration of cesium in the right ventricle and atrium is the same as that in the left ventricle, only the thicker left ventricular mass is detected by the scan. This myocardium is shown again in the isolated heart, emptied of blood, on the right. By spreading out the opened heart and scanning it we were able to confirm that the scan shows the thicker left ventricle and does not show the thinner right ventricle or atria. Myocardial infarcts were clearly shown as "cold" areas of decreased uptake. Fig. 4 shows a large anterior infarct. The decreased uptake in infarcts was confirmed by tissue counting. Since the experimental infarcts were al- 
ways in the anterior left ventricular wall, the posterior left ventricular wall (supplied by the unligated circumflex branch) served as a normal tissue for comparison. In 7 dogs the concentration of cesium-131 in samples of myocardium in which the infarction was confirmed by histologic examination was 25.1 per cent (range, 7.9 to 42.1 per cent) of that present in the normal left ventricular myocardium of the same dogs. The low uptake in infarcts was due to infarction and not to the fact that the tissues came from the anterior wall of the left ventricle, for in control dogs the concentration of cesium in the (normal) anterior left ventricular wall did not differ significantly from that in the normal posterior left ventricular wall. Sham-operated control dogs did not differ from unoperated control dogs either in scans or tissue counts.

In some dogs, tissue in the area supplied by the anterior descending branch of the left coronary artery failed to develop

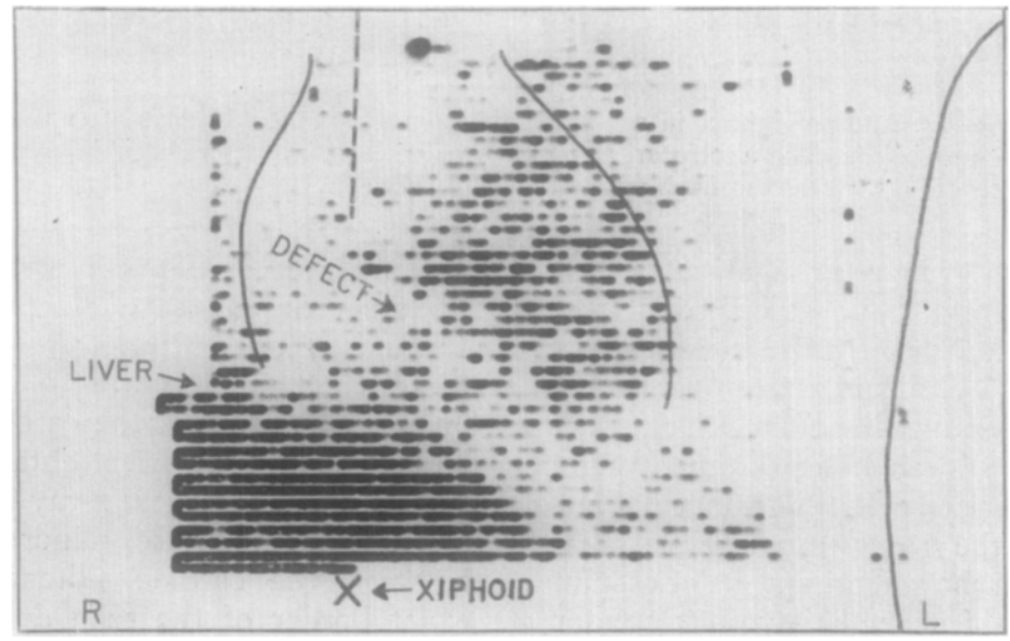

Fig. 9. Doubtful scan of a posterior infarct in a 50-year-old man scanned 10 days after the acute episode. The patient had no evidence of pericardial fluid, and a definite friction rub was audible at the time of scanning.

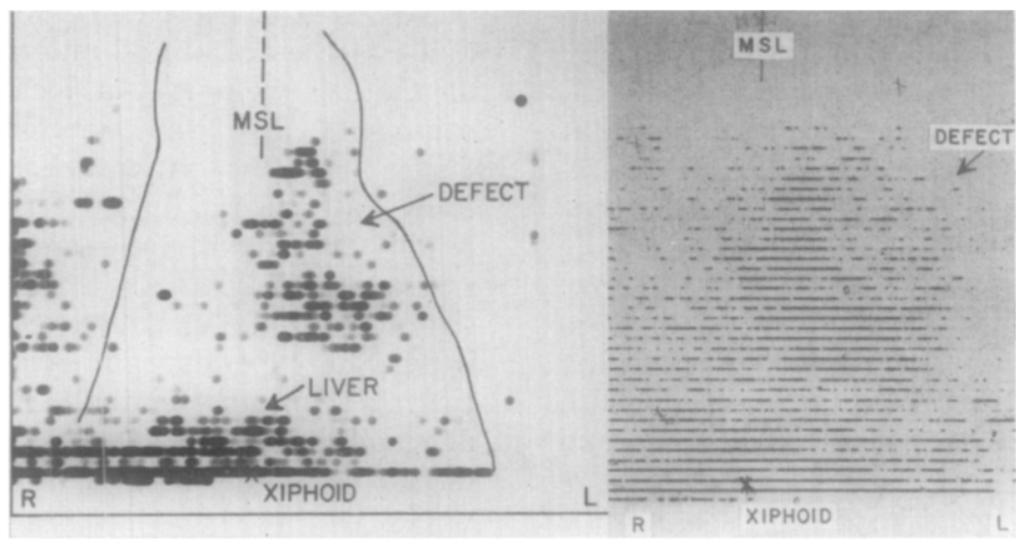

Fig. 10. High anterolateral infarct in a 51-year-old man, scanned 16 days after the acute episode. The photoscan on the left should be compared with the dot scan on the right. The separation between hepatic and myocardial densities represents pericardial fluid in this patient. The high lateral "cold" area of infarction is also clearly seen, as indicated by the arrow. A ventricular aneurysm was subsequently demonstrated by angiography in the latter area. 


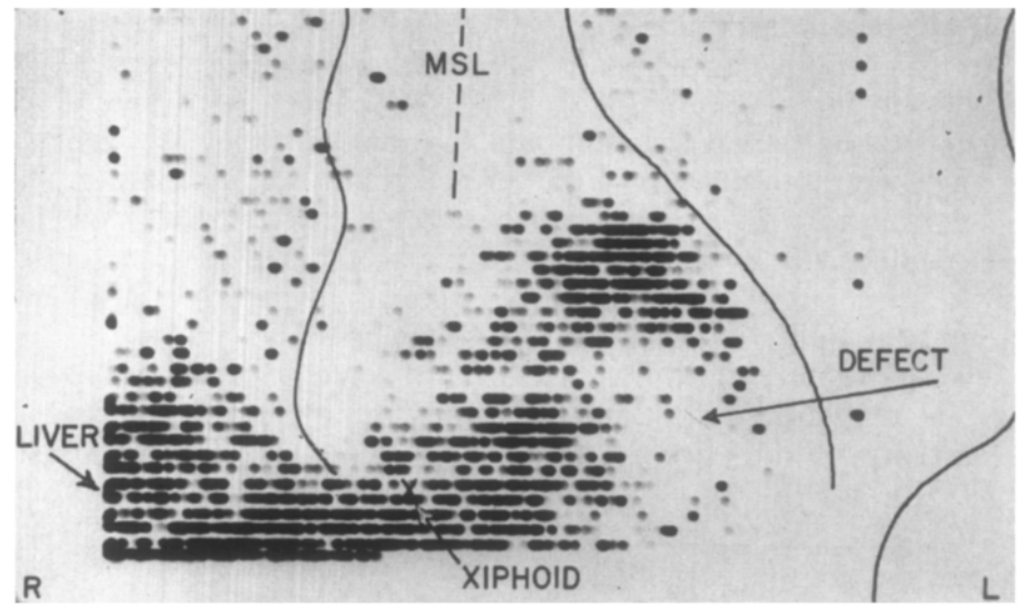

Fig. 11. Anterior infarct in a 68-year-old woman, scanned 14 days after the acute episode. The electrocardiogram showed evidence of a previous infarct but did not detect the fresh infarct.

frank necrosis, even after ligation of this artery. As previously described, ${ }^{2}$ such tissue has been arbitrarily classified as "ischemic." The mean concentration of radioactive cesium in such "ischemic" tissues in 6 dogs was 65.6 per cent (range, 19.7 to 99.4 per cent) of that in normal left ventricle of the same dogs. Thus, "ischemic" tissue showed an uptake of cesium less than that of normal tissue but greater than that of infarcted tissue.

Although our data do not yet permit a definite statement about the relationship between cesium uptake and the time that has elapsed since infarction, areas of infarction tended to have a very low concentration of cesium in dogs given the isotope 1 day after infarction, whereas there was a tendency toward somewhat higher concentrations in dogs which received cesium at later times. But the concentration of cesium in scar tissue in the 57-day-old infarct was also very low. Areas of "ischemia" showed low concentration when the isotope was given during the first few days after ligation, with fairly prompt recovery toward normal uptake by the fifth day.

Human beings. Fig. 5 shows a normal myocardial scan, obtained in a male control patient. The smooth, curved outline of the ventricular myocardium is apparent. The diaphragmatic surface of the heart is closely adjacent to the area of hepatic uptake. Fig. 6 shows another normal scan, obtained in a female control patient. Whereas our method of projecting the cardiac silhouette onto the scan in dogs should insure accurate placing of the outline on the scan, the cardiac silhouette in human beings was obtained from a separately taken chest $\mathrm{x}$-ray film, as noted above, and, therefore, minor discrepancies between the cardiac silhouette and the exact border of the scan density are not significant.

Figs. 7, 8, 9, and 10 show scans from patients who had previously been classified as having unequivocal fresh myocardial infarcts (see above). Fig. 7 shows a large "cold" area of decreased uptake involving much of the apical and anterolateral walls of the left ventricle, a location entirely consistent with the electrocardiographic findings in this patient. Fig. 8 shows a fresh anteroseptal infarct; the defect extends obliquely to the margin of the ventricular density. This location was consistent with the electrocardiographic findings. Our experience in dogs and in patients suggests that infarcts may be generally more easily detectable in the early days after infarction than during the second week. This fact may be significant in the scan shown in Fig. 9. This scan, taken 10 days after an acute posterior infarction (as located electrocardiographically), was read as doubtful. Although the crescentic area of decreased uptake in the diaphragmatic portion of the left ventricle, breaking 
the normal contour, suggests an infarct, and although this location is consistent with the electrocardiographic localization, the scan was not considered to be definitely positive. This scan was the only doubtful scan obtained in the 6 patients with unequivocal infarcts. The patient whose scan is shown in Fig. 9 had no evidence suggestive of pericardial effusion at the time of the scan, and, indeed, he had a marked friction rub at that time. Fig. 10 is a scan obtained in a patient with electrocardiographic evidence of a fresh, high anterolateral infarct. At the time of the scan, he was also known to have a pericardial effusion. An examination of the photoscan, shown on the left, and the dot scan, shown on the right, clearly reveals the defect due to the infarct, located high and laterally. The separation due to the accumulation of pericardial effusion causes a defect that is much less striking than one due to the infarct. Moreover, the relatively "cold" area between the heart and liver in this instance does not show any definite break in the smooth contour of the myocardium. This patient developed a ventricular aneurysm, as proved by angiography, in the area of recent infarction.

Of the 6 patients with unequivocal recent acute myocardial infarcts (Group a), the scans were positive in 5 and doubtful in 1 (Fig. 9). All 3 of the control patients (Group d) gave clearly negative scans.

Among the 4 patients in the two intermediate classes (Groups b and c) whose clinical status was not unequivocally established, the results were as follows. A patient who had had an anteroseptal infarct in 1961, and severe angina since January, 1963, was admitted to the University Hospital in March, 1963, with the clinical diagnosis of a fresh infarct. Serial changes in serum enzyme activities supported the clinical opinion and placed the date of the infarct about March 14. The electrocardiogram showed evidence of old infarction but no evidence of recent infarction. A scan taken on March 28, clearly showed a large anterior infarct (Fig. 11). In a second patient, a 56-year-old man with a history of old infarction, angina, and a recent clinical diagnosis of acute myocardial infarction, the clinical diagnosis was supported by changes in serum enzyme activities, but the electrocardiogram did not show diagnostic changes. The scan was read as negative. Thus, in the 2 patients who had probable but not certain acute infarcts (Group b) the scan was positive in one and negative in the other.

In Group c, a 55-year-old man had aortic stenosis, left ventricular hypertrophy, no history of angina, and no electrocardiographic evidence indicative of myocardial infarction. Cardiac catheterization and angiography had documented the aortic stenosis and had also shown stenosis of the left coronary artery without any evidence of complete occlusion. The scan was negative. The other patient in this group was a 72-year-old man with severe angina, hypertension, and congestive failure. Neither his electrocardiogram nor his history suggested any definite recent infarction. The scan was read as doubtful.

\section{Discussion}

Now that the use of cesium-131 has permitted successful visualization of infarcts in living patients, the possible uses of this technique may be considered. Accurate localization of infarcts by direct visualization is now a reasonable hope. Diagnosis of myocardial infarcts in patients with a doubtful history or electrocardiogram has probably been possible in at least one patient (Fig. 11). But we have deliberately chosen to evaluate the new technique chiefly by comparing unequivocal normal hearts with unequivocal infarcts. It was thought that this would lead to the most rapid determination of the validity of the test. Nevertheless, one is obviously more likely to turn to the scan in cases in which the clinical findings and electrocardiogram conflict than in cases in which there is no such conflict. Therefore, subsequent evaluation of myocardial scanning should probably include studies of doubtful cases, in which only necropsy findings will permit final evaluation.

We cannot yet say what value, if any, the scan will have in patients who are thought to have impending myocardial infarction or in the evaluation of patients with angina pectoris. We suspect that it will be more difficult to distinguish between the chronically ischemic myocar- 
dium and the myocardium with an acute infarct than to distinguish between the chronically ischemic myocardium and the normal myocardium. Thus, the scan may turn out to be useful in puzzling cases of severe heart pain. The possible usefulness of the myocardial scan in certain other disorders, e.g., myocarditis, remains to be evaluated. Although the suggested use of $I^{131}$ as iodide to demonstrate myocardial infarcts $^{5}$ proved to be disappointing, ${ }^{6,7}$ Evans $^{8}$ has indicated the feasibility of demonstrating infarcts by heart scans after the administration of radioiodinated fatty acids; this interesting approach also deserves to be evaluated in disorders such as myocarditis.

The scan might theoretically offer the possibility of directly showing the thickness of the left ventricular wall, but one must be cautious in this regard. The technique of myocardial scanning has been deliberately arranged in such a way as to produce a full even density in the normal heart, without showing the left ventricular cavity. If one changes the technique in order to bring the left ventricular cavity into focus, then one has the possibility of misinterpreting the left ventricular cavity as a "cold" area of infarction. With proper attention to the setting of the scanner, it has been found quite easy to avoid mistaking the normal left ventricular cavity for a "cold" area, especially if one remembers that large infarcts tend to reach the outer surface of the myocardium in one or more areas of the scan. This conservative method of reading is recommended in order to prevent false-positive diagnoses of myocardial infarction. It is admitted that such conservatism will probably sacrifice our ability to detect left ventricular hypertrophy, and, quite possibly, our ability to detect subendocardial infarcts.

\section{Summary}

1. It is possible to visualize the left ventricular myocardium of the intact, living patient, without discomfort or danger to the patient, by photoscanning after the administration of cesium-131.

2. The normal left ventricular myocardium appears as a full, even density, with a smooth contour. Myocardial infarcts appear as "cold" areas of decreased uptake.

3. In 6 patients with unequivocal fresh myocardial infarcts, the scans were positive in 5 and doubtful in 1 . In 2 patients who probably had suffered recent myocardial infarctions, the scan was positive in 1 and negative in 1 . In 2 patients who had heart disease but who had probably not had recent myocardial infarctions, the scan was negative in 1 and doubtful in 1 . The scan was negative in each of 3 patients with normal hearts.

\section{REFERENCES}

1. Carr, E. A., Jr., Beierwaltes, W. H., Wegst, A. V., and Bartlett, J. D., Jr.: Myocardial scanning with rubidium-86, J. Nuclear Med. $3: 76,1962$.

2. Carr, E. A., Jr., Beierwaltes, W. H., Patno, M. E., Bartlett, J. D., Jr., and Wegst, A. V.: The detection of experimental myocardial infarcts by photoscanning, AM. HEART J. 64:650, 1962.

3. Carr, E. A., Jr., Cafruny, E. J., Beierwaltes, W. H., and Bartlett, J. D., Jr.: Evaluation of $\mathrm{Hg}^{203}$-chlormerodrin in the demonstration of human myocardial infarcts by scanning, University of Michigan Med. Bull. 1:27, 1963.

4. Levy, M. N., and Cutarelli, R.: Effect of acute coronary artery ligation upon blood flow to the normal myocardium, Am. J. Cardiol. 13:48, 1964.

5. Dreyfuss, F., Ben-Porath, M., and Menczel, J.: Radioiodine uptake in the infarcted heart, Am. J. Cardiol. 6:237, 1960.

6. Lourvanis, B. E., and Silberstein, H. E.: Failure of in vivo detection of $\mathrm{I}^{131}$ localization in cardiac infarcts of dogs, Mt. Sinai Hosp. J., Baltimore 8:153, 1959

7. Mason, D. T., Fry, R. L., and Wagner, H. N., Jr.: Radioisotope scanning of the precordial distribution of iodide in patients with myocardial infarction, Circulation 24:1338, 1961.

8. Evans, R. J., Gunton, R. W., Beanlands, D. S., and Baker, R. G.: Photoscans of the heart with radioiodinated fatty acid (R.I.F.A.), (Abstract) Canad. M.A.J. 88:256, 1963. 